\title{
Fighting against intracellular pathogens: host cell-targeted drug delivery
}

\author{
Zsuzsa Baranyai*,1 iD \\ ${ }^{1}$ Instituto de Ciencia de Materiales de Aragón (ICMA) - Consejo Superior de Investigaciones Científicas (CSIC)/Universidad de \\ Zaragoza, Edificio I+D+i - Campus Río Ebro, C/ Mariano Esquillor s/n Zaragoza 50018, Spain \\ *Author for correspondence: zsuzsabaranyai@unizar.es
}

\begin{abstract}
"Nanomedicine with active targeting strategies is one of the most promising approaches to ensure that drug molecules reach their target sites and it brings us closer to eradicating harmful intracellular pathogens."
\end{abstract}

First draft submitted: 21 February 2020; Accepted for publication: 20 May 2020; Published online: 13 July 2020

Keywords: intracellular pathogens $\bullet$ macrophage targeting $\bullet$ nanocarriers $\bullet$ passive $\&$ active targeting

Infectious diseases caused by diverse intracellular pathogens, such as viruses, some bacteria, fungi and protozoan parasites are major health threats worldwide. Particularly, Mycobacterium tuberculosis, Plasmodium parasites and HIV, causative agents of tuberculosis (TB), malaria and AIDS, respectively, infect more than a quarter of the world's population resulting more than two million deaths per year [1-3]. Moreover, numerous other intracellular pathogens such as Leishmania parasites, Salmonella enterica, Listeria monocytogenes, Neisseria meningitidis, Chlamydia trachomatis and viruses also exhibit serious health risks. In addition, it is increasingly recognized that many bacteria that were considered extracellular can also thrive or persist inside cells [4]. Intracellular pathogens can avoid elimination by the host immune system using various escape mechanisms and they can establish a persistent infection [5]. The treatment of these diseases is challenging due to the limitations of efficient drug transport into the host cells. These infections are usually treated with high doses of antimicrobial agents for a longer period that can be accompanied by serious side effects and the risk of development of drug resistance. To overcome these challenges there is a need to develop strategies that can ensure that the therapeutic compounds reach their target sites.

Many microbes developed successful strategies to invade the host while evading its immunity. Surprisingly, several pathogens chose an extreme environment to live in: the mononuclear phagocytes [5,6]. Based on this, a key target for drug delivery against the majority of intracellular pathogens are the mononuclear phagocytes. The cells of the mononuclear phagocyte system (MPS), such as monocytes, macrophages and dendritic cells, are the most efficient cell types of antimicrobial defence. In some cases, neutrophils, fibroblasts or epithelial cells can also serve as habitat for intracellular pathogens. The majority of intracellular bacteria remains in endocytic or phagocytic vacuoles of the host cells, which they reprogram to provide an ideal environment for their survival, while others enter the cytosol $[4,5]$.

To reach the reservoirs of intracellular pathogens, a variety of nanocarriers have been developed. Polymeric nanoparticles, nanocapsules, micelles, dendrimers, nanogels, liposomes, solid-lipid nanoparticles, inorganic nanocarriers, etc. were introduced as promising drug delivery systems. Antimicrobial agents can be loaded into nanocarriers either by physical encapsulation, adsorption or chemical conjugation. Main advantages of nanocarrier systems as compared with free drugs are enhanced bioavailability, protection of the entrapped drug from inactivation, controlled drug release, the possibility to reduce the administered doses and thus the related toxic side effects and the frequency of administration. Importantly, using nanocarriers, it is possible to target host cells or infection site by passive accumulation or by active targeting using specific ligands $[7,8]$.

It is a prominent choice to passively target the host cells in the MPS by nanocarriers due to the natural propensity of these cells to phagocyte particles. Moreover, nanocarriers can be tailored for enhanced uptake by MPS varying their characteristics such as size, charge, rigidness or shape. Opsonization also promotes phagocytosis by MPS. The rapid accumulation in the MPS of nanocarriers against intracellular pathogens is an advantage, while 
in case of nanocarriers aiming tumors, it is a disadvantage. The infection site can also be passively targeted by nanocarriers due to the enhanced permeation and retention effect, resulting in the microvascular permeability of the capillaries becoming locally increased and the lymphatic drainage becoming impaired due to inflammatory reactions $[9,10]$. Plenty of studies demonstrate that using nanocarriers, enhanced in vitro and in vivo bioavailability was achieved $[7,8,11,12]$.

The specificity of drug-loaded nanocarriers can be significantly enhanced with ligand-receptor targeting strategy as an active targeting approach, by taking advantage of characteristic receptors of MPS cells. To create specific nanocarriers to host cells, different targeting molecules can be introduced onto their surface. Such targeting ligands can be, among others, proteins, antibodies, peptides and carbohydrates [13]. Ligands can be designed to be similar to conserved structures of microbial pathogens called pathogen-associated molecular patterns (PAMPs) and, therefore, can be recognized by the pattern recognition receptors (PRRs) of phagocytes. One main group of membrane-bound PPRs are the toll-like receptors, which recognize mainly glycoproteins, lipoproteins, heat shock proteins and flagellar proteins. Another main group is the C-type lectin receptors with specificity for carbohydrate derivatives expressed on the surface of microbes (e.g., mannose, galactose, fucose, glycolipids) [14]. The scavenger receptor superfamily, a subset of membrane-bound PPRs, recognizes various ligands including polyionic molecules [15].

Besides being common host cells for intracellular pathogens, macrophages are of particular interest as target cells due to their extensive involvement in other high-impact diseases, such as cancer and inflammatory diseases [9,16]. However, recent reviews discussing especially the achievements of active targeting of macrophages in case of intracellular infections are lacking. Hereafter, some current examples of this wide area are highlighted.

Many studies have focused on targeting macrophages through their mannose receptor (CD206), a C-type lectin receptor that can recognize ligands with a terminal mannose, $N$-acetylglucosamine or fucose moiety [17]. Different mannosylated carriers showed enhanced cellular uptake by macrophages [12]. Vancomycin-loaded mannosylated nanogels were active against MRSA infected macrophages and could effectively deliver vancomicyn to the bacterial infection site and kill the bacteria in a zebrafish embryo in vivo model [18]. Inhalable, rifampicin-loaded mannosylated solid lipid nanoparticles were developed to target alveolar macrophages in TB therapy [19]. Isoniazid-loaded mannosylated gelatin nanoparticles reduced the bacterial counts in the lungs and spleen of TB-infected mice accompanied by reduced hepatotoxicity [20]. Besides targeting, mannosylation of chitosan nanocarriers remodeled the macrophage response to bacterial infection, in particular affecting the regulation of many metabolic pathways [21].

Folate receptors that are overexpressed on the surface of activated macrophages also have been used for targeting. Folic acid decorated antiretroviral drug nanocrystals displayed enhanced uptake by macrophages compared with nontargeted particles [22]. In a murine model of pulmonary Pseudomonas aeruginosa infection, folic acid-modified moxifloxacin-loaded $\alpha$-cyclodextrin based nanoparticles showed better antibacterial efficacy with a prolonged survival time than free moxifloxacin and nontargeted nanoparticles [23].

Hyaluronic acid receptors on macrophages, such as CD44 can also serve as targets. Hyaluronic acid-tocopherol succinate micelles enhanced the in vitro uptake of the incorporated rifampicin in alveolar macrophages [24]. Respirable particles were prepared from nanosuspension of sodium hyaluronate, rifampicin, isoniazid and verapamil, and showed intracellular antitubercular activity on infected macrophages [25].

Tuftsin, a natural immunostimulatory tetrapeptide, has been reported as a macrophage-targeting and stimulating peptide. Grafting of tuftsin on liposome surface was used successfully in vivo in different macrophage-based infections [26]. Decorating poly(D,L-lactic-co-glycolic acid) nanoparticles with tuftsin derivatives increased the internalization rate and the intracellular activity of the encapsulated drug candidate against $M$. tuberculosis in vitro [27].

Formyl peptide receptors (FPRs) transduce chemotactic signals in phagocytes, mediate host-defense and inflammatory responses. FPRs recognize peptides containing $N$-formylated methionine (e.g., fMLF peptide), such as cleavage products of bacterial and mitochondrial proteins, therefore considered as pattern recognition receptors [28]. Poly(ethylene glycol) nanocarriers conjugated with $N$-formylmethionyl-leucyl-phenylalanine (fMLF) peptide showed greater in vivo accumulation into peritoneal macrophages than unmodified poly(ethylene glycol) [29]. However, the area of targeting the MPS with FPR ligand modified nanocarriers is far from being exploited.

Internalization of nanocarriers by MPS host cells follows similar routes as internalization of pathogens, mainly through phagocytosis or endocytosis. Once internalized, nanocarriers are located in phagosomes or endosomes, and after maturation and fusion processes they can end up in lysosomes. In these acidic compartments, nanocarriers can undergo degradation leading to releasing their antimicrobial cargo and reaching, as well as eliminating the pathogen 
within the same vesicle. Moreover, diffusible drugs can escape from the endolysosomes to reach pathogens located in the cytosol or other subcellular compartments [7].

Nanomedicine with active targeting strategies is one of the most promising approaches to ensure that drug molecules reach their target sites and it brings us closer to eradicating harmful intracellular pathogens. However, the use of nanocarriers also faces challenges, such as stability, reproducibility, possible toxicity and cost-effectiveness issues. Several nanomedicine formulations have been approved for clinical use so far, however, among them there are only a few used against infections caused by intracellular pathogens [30]. Further research needs to be oriented toward creating and studying highly effective targeted nanocarriers, and to understanding the complex mechanisms of the interaction between nanocarriers, host cells and intracellular pathogens. Such efforts undoubtedly can bring impressive developments in this field.

\section{Financial \& competing interests disclosure}

This work has been supported by the European Union's Horizon 2020 research and innovation programme under the Marie Sklodowska-Curie grant agreement No 842652, by the Spanish MICINN project BIO2017-84246-C2-1-R and by Gobierno de Aragón (Diputación General de Aragón-Fondo Social Europeo).

The author has no other relevant affiliations or financial involvement with any organization or entity with a financial interest in or financial conflict with the subject matter or materials discussed in the manuscript apart from those disclosed.

No writing assistance was utilized in the production of this manuscript.

\section{References}

1. WHO. WHO Global tuberculosis report 2019.

https://apps.who.int/iris/bitstream/handle/10665/329368/9789241565714-eng.pdf?ua=1

2. WHO. World malaria report 2019. https://apps.who.int/iris/rest/bitstreams/1262394/retrieve

3. WHO. Progress report on HIV, viral hepatitis and sexually transmitted infections 2019. https://apps.who.int/iris/bitstream/handle/10665/324797/WHO-CDS-HIV-19.7-eng.pdf?ua=1

4. Cossart P, Helenius A. Endocytosis of viruses and bacteria. Cold Spring Harb. Perspect. Biol. 6(8), a016972 (2014).

5. Thakur A, Mikkelsen H, Jungersen G. Intracellular pathogens: host immunity and microbial persistence strategies. J. Immunol. Res. 2019, 1356540 (2019).

6. Kaufmann SH. Intracellular pathogens: living in an extreme environment. Immunol. Rev. 240(1), 5-10 (2011).

7. Ladavière C, Gref R. Toward an optimized treatment of intracellular bacterial infections: input of nanoparticulate drug delivery systems. Nanomedicine (Lond). 10(19), 3033-3055 (2015).

8. Atbiaw N, Aman E, Dessalegn B et al. Review on targeted drug delivery against intracellular pathogen. Pharm. Pharmacol. Int. J. 6(3), 183-189 (2018).

9. Hu G, Guo M, Xu J et al. Nanoparticles targeting macrophages as potential clinical therapeutic agents against cancer and inflammation. Front. Immunol. 10, 1998 (2019).

10. Azzopardi EA, Ferguson EL, Thomas DW. The enhanced permeability retention effect: a new paradigm for drug targeting in infection. J. Antimicrob. Chemother. 68(2), 257-274 (2013).

11. Griffiths G, Nyström B, Sable SB, Khuller GK. Nanobead-based interventions for the treatment and prevention of tuberculosis. Nat. Rev. Microbiol. 8(11), 827-834 (2010).

12. Grotz E, Tateosian N, Amiano N et al. Nanotechnology in tuberculosis: state of the art and the challenges ahead. Pharm. Res. 35(11), 213 (2018).

13. Lin YS, Lee MY, Yang CH, Huang KS. Active targeted drug delivery for microbes using nano-carriers. Curr. Top. Med. Chem. 15(15), 1525-1531 (2015).

14. Takeuchi O, Akira S. Pattern recognition receptors and inflammation. Cell 140(6), 805-820 (2010).

15. Zani IA, Stephen SL, Mughal NA et al. Scavenger receptor structure and function in health and disease. Cells 4(2), 178-201 (2015).

16. Ponzoni M, Pastorino F, Di Paolo D, Perri P, Brignole C. Targeting macrophages as a potential therapeutic intervention: impact on inflammatory diseases and cancer. Int. J. Mol. Sci. 19(7), E1953 (2018).

17. Azad AK, Rajaram MV, Schlesinger LS. Exploitation of the macrophage mannose receptor (CD206) in infectious disease diagnostics and therapeutics. J. Cytol. Mol. Biol. 1(1), 1000003 (2014).

18. Xiong MH, Li YJ, Bao Y, Yang XZ, Hu B, Wang J. Bacteria-responsive multifunctional nanogel for targeted antibiotic delivery. Adv. Mater. 24(46), 6175-6180 (2012).

19. Maretti E, Costantino L, Buttini F et al. Newly synthesized surfactants for surface mannosylation of respirable SLN assemblies to target macrophages in tuberculosis therapy. Drug Deliv. Transl. Res. 9(1), 298-310 (2019). 
20. Saraogi GK, Sharma B, Joshi B et al. Mannosylated gelatin nanoparticles bearing isoniazid for effective management of tuberculosis. J. Drug Target. 19(3), 219-227 (2011).

21. Coya JM, De Matteis L, Giraud-Gatineau A et al. Tri-mannose grafting of chitosan nanocarriers remodels the macrophage response to bacterial infection. J. Nanobiotechnol. 17(1), 15 (2019).

22. Zhou T, Lin Z, Puligujja P et al. Optimizing the preparation and stability of decorated antiretroviral drug nanocrystals. Nanomedicine (Lond). 13(8), 871-885 (2018).

23. Wang Y, Yuan Q, Feng W et al. Targeted delivery of antibiotics to the infected pulmonary tissues using ROS-responsive nanoparticles. J. Nanobiotechnol. 17(1), 103 (2019).

24. Gao Y, Sarfraz MK, Clas SD, Roa W, Löbenberg R. Hyaluronic acid-tocopherol succinate-based self-assembling micelles for targeted delivery of rifampicin to alveolar macrophages. J. Biomed. Nanotechnol. 11(8), 1312-1329 (2015).

25. Rossi I, Buttini F, Sonvico F et al. Sodium hyaluronate nanocomposite respirable microparticles to tackle antibiotic resistance with potential application in treatment of mycobacterial pulmonary infections. Pharmaceutics 11(5), E203 (2019).

26. Agrawal AK, Gupta CM. Tuftsin-bearing liposomes in treatment of macrophage-based infections. Adv. Drug Deliv. Rev. 41, 135-146 (2000).

27. Horváti K, Gyulai G, Csámpai A, Rohonczy J, Kiss É, Bősze S. Surface layer modification of poly(D,L-lactic-co-glycolic acid) nanoparticles with targeting peptide: a convenient synthetic route for pluronic F127-tuftsin conjugate. Bioconjug. Chem. 29(5), 1495-1499 (2018).

28. He HQ, Ye RD. Molecules. The formyl peptide receptors: diversity of ligands and mechanism for recognition. Molecules 22(3), E455 (2017).

29. Gunaseelan S, Gunaseelan K, Deshmukh M, Zhang X, Sinko PJ. Surface modifications of nanocarriers for effective intracellular delivery of anti-HIV drugs. Adv. Drug. Deliv. Rev. 62(4-5), 518-531 (2010).

30. Ventola CL. Progress in nanomedicine: approved and investigational nanodrugs. Pharm. Ther. 42(12), 742-755 (2017). 